\title{
Therapeutic challenge: severe dyspnea in a patient with metastatic breast cancer and lymphangitis carcinomatosis syndrome
}

\section{Abstract}

Introduction. Dyspnea, regardless of etiology (pulmonary, neuromuscular or caused by other factors), remains a significant challenge. There is still a lack of effective methods of the treatment of severe dyspnea associated with intensifying anxiety which is the most common indication for intensification of causative and symptomatic treatment or palliative sedation.

Case report. A 48 year-old woman diagnosed with relapsed breast cancer with bone, pleural, hepatic and pulmonary metastases with lymphangitis carcinomatosis syndrome confirmed by lung biopsy. In 2011 the patient underwent breast conserving surgery followed by teletherapy, brachytherapy and tamoxifen hormone therapy for 5 years. Comorbidities included chronic obstructive pulmonary disease, diabetes mellitus type 2, depression and nicotine addiction.

The patient was urgently admitted to the Department of Oncology and Radiotherapy from Clinical Emergency Department due to rapidly aggravating dyspnea and respiratory failure. Chest X-ray showed massive bilateral pleural effusion and threatening cardiac tamponade. Treatment involved thoracentesis and pericardiocentesis with concurrent symptomatic management. After temporary stabilization of the patient and due to rapid progression of the disease salvage, paclitaxel chemotherapy was started. Despite the features of potential chemosensitivity (chemotherapy-naïve, rapid progression, visceral metastases) the applied treatment was ineffective. After careful evaluation of clinical situation and possible treatment strategies, and after obtaining patient's consent, sedation with midazolam and morphine was applied. Conclusions. This case demonstrated ineffective salvage chemotherapy used in the patient with severe dyspnea induced by a rapid progression of metastatic breast cancer and difficulties in obtaining effective symptomatic treatment.

$$
\text { Palliat Med Pract 2019; 13, 3: 156-160 }
$$

Key words: dyspnea, lymphangitis carcinomatosis, symptomatic treatment, salvage chemotherapy

\section{Introduction}

Dyspnea is often described by patients as suffocating, choking or shortness of breath [1].In cancer patients, it can be caused by different mechanisms such as airways obstruction, loss of functional pulmo- nary tissue (for example due to multiple metastases), presence of fluid - both in bronchoalveolar tract and in pleural cavity, pulmonary embolism or as a consequence of lymphangitis carcinomatosis syndrome. Breathlessness exerts a major impact on patients' and caregivers' quality of life and generates higher distress

\footnotetext{
Address for correspondence:

Konrad Gądek

Gdański Uniwersytet Medyczny

ul. Marii Skłodowskiej-Curie 3A

80-210 Gdańsk

tel.: 504412156

e-mail: kgadek@gumed.edu.pl 
than pain or fatigue [1]. We present a patient with severe breathlessness and accompanying lymphangitis carcinomatosis. The first female patient with disseminated uterus cancer was depicted by French pathologist Gabriel Andral in 1824, followed by Virchow in 1855, Bristowe in 1868, a Reynaud in 1874 [2] and the term lymphangitis carcinomatosis was introduced by Troisier in 1873 to describe diffuse infiltration of the lymphatics of both the lungs by malignant cells [3]. In most cases this clinical condition is related to variety of symptoms, with dominating occurrence of dyspnea [4].

\section{The case depiction}

A 48 year-old woman with luminal A breast cancer, in 2011 patient underwent breast conserving surgery followed by teletherapy, brachytherapy and tamoxifen hormonotherapy. Comorbidities included chronic obstructive pulmonary disease (COPD) stage 3 category B GOLD classification, diabetes mellitus type 2 , and depression. The patient was also a former smoker.

First symptoms of cancer relapse were fatigue, slow progression of dyspnea and condition deterioration that occurred 3 months before respiratory failure. At that moment the patient was admitted to Pneumonology Department and the COPD deterioration was suspected. It should be pointed that patient's general condition was good according to Eastern Cooperative Oncology Group (ECOG) 0 across previous years with the excellent control of all comorbidities. After chest computed tomography (Fig. 1-3) and spirometry the initial diagnosis was rejected. Results shown multiple pulmonary, pleural, hepatic, and bone metastases. The lung biopsy was performed and histopathological diagnosis of lymphangitis carcinomatosis (Fig. $5 \mathrm{~A}$ and B) was established. Additional immunochemistry was performed which confirmed relapse of breast cancer.

At the time of relapse, the patient encountered acute stress reaction, confirmed by psychiatrist consultation. Adequate treatment with sertraline, diazepam and psychological support was introduced; however, the expected effects were not obtained. Initially, the patient refused any anticancer treatment due to poor psychological condition.

Twelve weeks from the diagnosis of recurrence the patient was admitted to the Clinical Emergency Department with severe dyspnea and respiratory failure. Performance status according to ECOG was assessed as 4. Physical examination confirmed tachypnoea, tachycardia, and hypertension associated with dyspnea and anxiety. Auscultation of lungs revealed bilateral muting over the lower pulmonary fields and

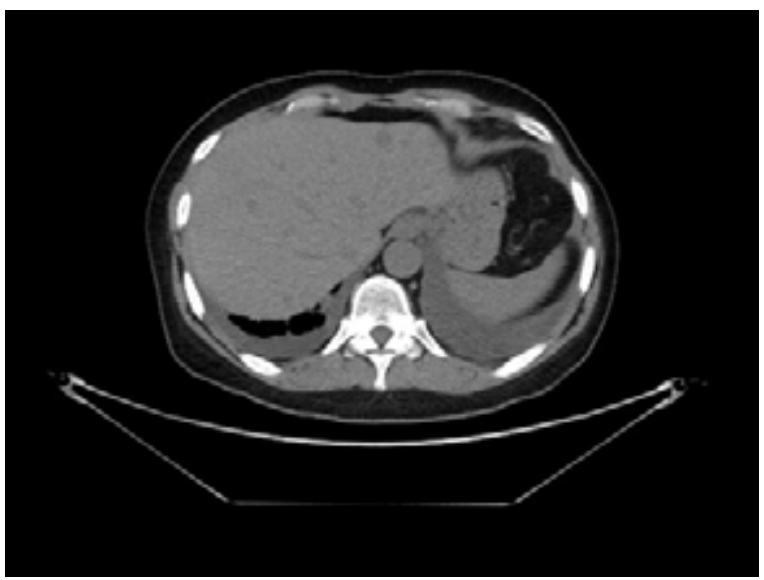

Figure 1. Computed Tomography (CT) scan showing liver metastases

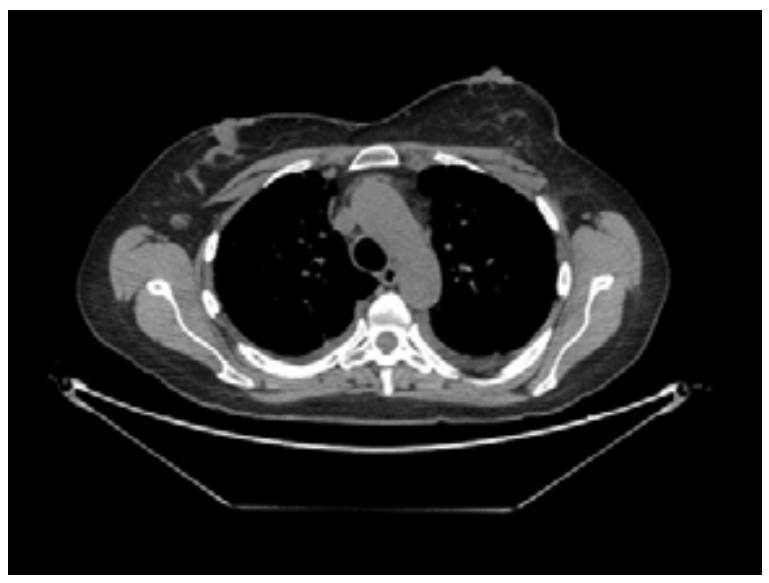

Figure 2. CT scan - local recurrence in the site of breast amputation

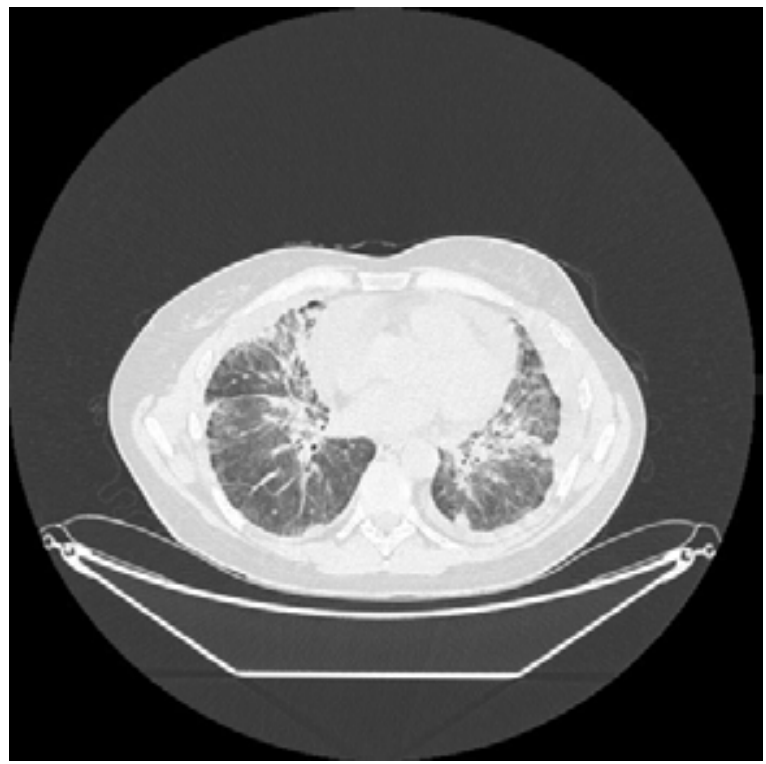

Figure 3. CT scan - visible characteristic presentation of lymphangitis carcinomatosa syndrome - 'tree in bud' 


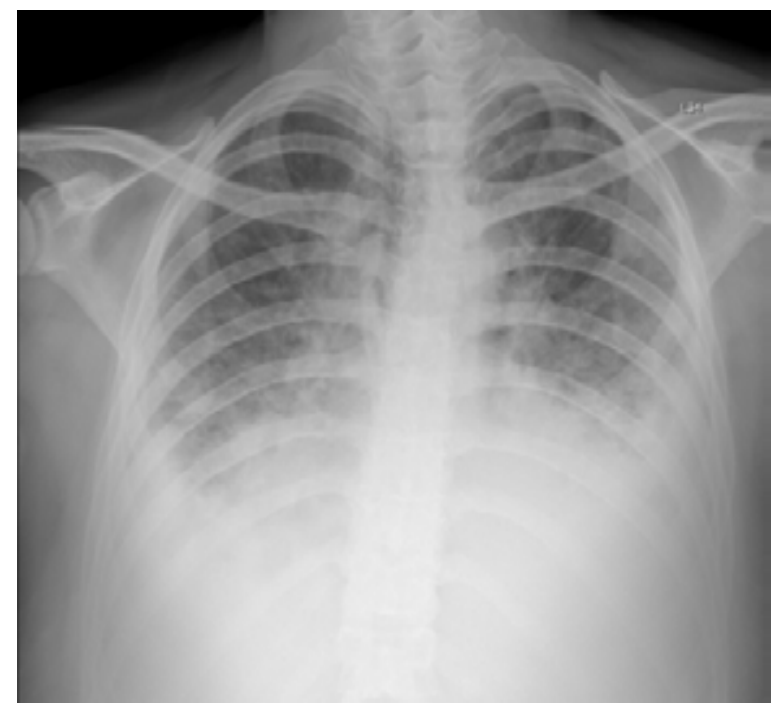

Figure 4. Chest X-Ray - pulmonary edema

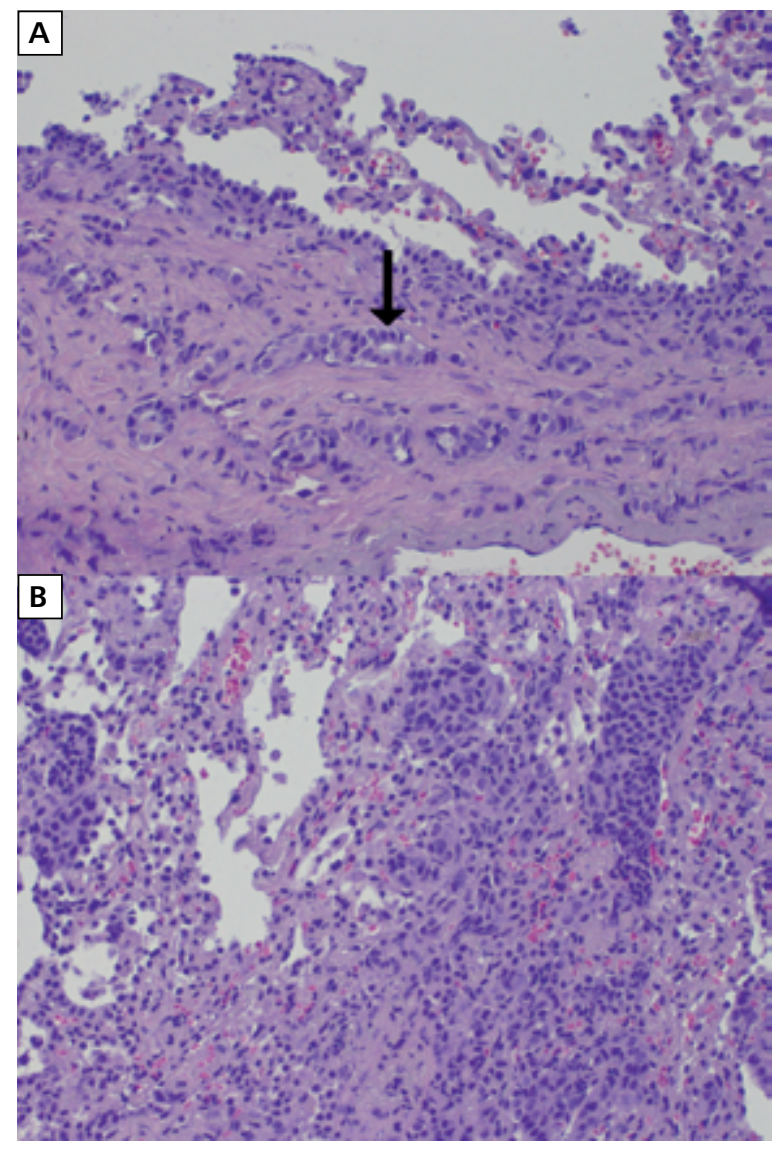

Figure 5 A-B. histopathological samples acquired from lung - HE stain - visible cancer cells in lung capillaries

bilateral crackles. Chest X-ray (Fig. 4) revealed bilateral hydrothorax to the level of IV rib in the anterior orientation. In comparison with an X-ray conducted few days earlier (by patient's family doctor) the fluid levels significantly increased in both pleural cavities. The V-Scan ultrasonography showed significant amount of fluid in pericardial sac, up to $24 \mathrm{~mm}$ behind right ventricle and right atrium; yet, still without the clinical manifestation of tamponade. The patient underwent right thoracentesis under local anaesthesia. Six hundred $\mathrm{mL}$ of bloody tinted fluid was evacuated and further drainage was planned. The procedure was complicated by clinically insignificant pneumothorax.

The next day patient was moved to Oncology and Radiotherapy Department for symptomatic and (if possible) palliative anticancer treatment. Symptomatic treatment consisted of oxygen, corticosteroids and betamimetics in nebulization, prednisone or dexamethasone orally, acetylcysteine intravenously (IV). The pain was effectively managed with paracetamol IV, morphine administered subcutaneously (SC) as needed and transdermal buprenorphine. Additionally, the patient received furosemide and amoxicillin/clavulanic acid IV, and pantoprazole orally. Unfortunately, further escalation of symptoms and poor overall status indicated that hydrothorax was not an individual cause of dyspnea and deterioration of performance status.

Heart function reassessment with V-scan ultrasonography was conducted and clinically significant pericardial tamponade was revealed; hence, the patient was transferred to Cardio-Surgery Department for additional procedures. Pericardiocentesis and left pleural cavity drainage were conducted which partially alleviate the symptoms. Overall status was stabilized (ECOG 3) for a few days. Although a slight improvement in symptom intensity was observed, further treatment was still required.

Four days after cardiosurgical intervention salvage chemotherapy was started in accordance with the oncological board decision. This decision was based on the clinical factors that suggested potential chemosensitivity of cancer, patients age and will. The first cycle of paclitaxel chemotherapy (total dose per cycle $100 \mathrm{mg}$ ) was administered along with premedication including dexamethasone, clemastine, and ondansetron. While early tolerance of chemotherapy was good, further days showed that the treatment was ineffective, and the patient's general status deteriorated quickly. Symptom burden, especially intense dyspnea, were unacceptable and lead to the extremely high level of anxiety, as the patient was fully conscious and aware of impending death. With the support of clinical psychologist possible treatment was discussed and the options were proposed to the patient and her family. Sedation has been chosen and informed consent to the procedures was given by the patient.

Morphine and midazolam in continuous intravenous infusion were used with the expected positive 
effect. Shortly after infusion began, patient was mostly unconscious with the moments of shallow sleep. The level of anxiety was reduced and patient remained calm. One day later the patient died in her sleep, primary cause of death was respiratory failure.

\section{Discussion}

Dyspnea remains a significant challenge in palliative and supportive care; hence, there is still a need to find a new or improve the existing ways to alleviate this symptom. In compare to the treatment of pain, breathlessness therapy remains limited. The clinicians are using a few effective groups of drugs, from which opioids and benzodiazepines play a crucial role when all other treatment methods are exhausted [1]. In the presented patient the treatment schedule included differential diagnosis, as the dyspnea deterioration had multiple origins: mechanical restriction of lungs by pleural fluid and metastases, cardiogenic - caused by heart tamponade, potential obturation due to COPD exacerbation, and lymphangitis carcinomatosis syndrome [5]. The multidisciplinary board made the difficult decision of aggressive treatment due to few important clinical factors that suggested potential chemosensitivity: chemo-naïve cancer, rapid progression of the disease (suggest high mitotic index), good performance status shortly before cancer relapse and young age of the patient $[6,7]$.

Nevertheless, to start cytostatic treatment patient's general status had to be stabilized, thus multiple invasive procedures were conducted. The presented case addresses therapeutic challenges occurring when different causes of dyspnea coexist. However almost all mentioned causes were induced by cancer progression. The oncological board decided to start salvage chemotherapy, which was at high risk due to poor general condition of the patient. Cytostatic treatment is a gold standard in the chemotherapy-naïve metastatic breast cancer, particularly in symptomatic pulmonary metastases or lymphangitis carcinomatosis syndrome. Unfortunately, treatment failed to reach it's goals: alleviation of symptoms and further stabilization of the disease, despite the potentially chemosensitive tumour.

Lymphangitis carcinomatosis is a rare clinical state in which the malignant cells are infiltrating the lymphatics. The appearance of the syndrome has been reported in skin [8], duodenum [9] and lungs [4]. In the pulmonary manifestation the most predominant symptom is intense breathlessness that cannot be relieved by standard procedures [4]. Primary tumours are typically adenocarcinoma located most commonly in breast, stomach, colon, pancreas, thyroid, cervix and prostate. Clinical diagnosis is currently based on the chest X-ray and High-Resolution Computed Tomography often confirmed by histopathological examination of biopsy-derived tissue [10]. In addition to symptomatic management standard treatment relies on chemotherapy [11], which is usually the only causative treatment ${ }^{3}$. It should also be noted that this type of patient is often in poor performance status with poor prognosis and choice of chemotherapy regimen might be challenging.

This patient was provided with the multidisciplinary team care. The holistic approach gave the opportunity for adequate treatment both causative and symptomatic. In the case of chemotherapy-naïve and chemotherapy sensitive advance cancer this treatment type gives the best results, even in patients with poor performance status. Based on available clinical data it was assumed the potentially high chemosensitivity of cancer. Unfortunately, the applied treatment was ineffective probably due to late implementation of individual cytostatic resistance. After exhausting all therapeutic options palliative sedation was instituted due to the intense shortness of breath and severe anxiety, which are the most common indications for this procedure [3].

\section{References}

1. Tishelman C, Degner LF, Rudman A, et al. Symptoms in patients with lung carcinoma: distinguishing distress from intensity. Cancer. 2005; 104(9): 2013-2021, doi: 10.1002/cncr.21398, indexed in Pubmed: 16178002.

2. Doyle L. Gabriel Andral (1797-1876) and the first reports of lymphangitis carcinomatosa. J R Soc Med. 1989; 82(8): 491-493, indexed in Pubmed: 2674433.

3. Bruce DM, Heys SD, Eremin O. Lymphangitis carcinomatosa: a literature review. J R Coll Surg Edinb. 1996; 41(1): 7-13, indexed in Pubmed: 8930034.

4. Mendeloff Al. Severe asthamatic dyspnoea as the sole presenting symptom of generalized endolymphatic carcinomatosis. Ann Intern Med. 1951; 34: 881-888.

5. Berliner D, Schneider N, Welte T, et al. The Differential Diagnosis of Dyspnea. Dtsch Arztebl Int. 2016; 113(49): 834-845, doi: 10.3238/arztebl.2016.0834, indexed in Pubmed: 28098068.

6. Klimek M. Pulmonary lymphangitis carcinomatosis: systematic review and meta-analysis of case reports, 1970-2018. Postgrad Med. 2019; 131(5): 309-318, doi: 10.1080/00325481.2019.1595982, indexed in Pubmed: 30900501.

7. Yu Z, Sankar S, Huben M. Excellent Response with Ado-Trastuzumab Emtansine in a Patient with Relapsed Metastatic Breast Cancer Presenting with Pulmonary Lymphangitic Carcinomatosis. Cureus. 2017; 9(7): e1473, doi: 10.7759/cureus.1473, indexed in Pubmed: 28944112.

8. Damstra RJ, Jagtman EA, Steijlen PM. Cancer-related secondary lymphoedema due to cutaneous lymphangitis carcinomatosa: clinical presentations and review of litera- 
ture. Eur J Cancer Care (Engl). 2010; 19(5): 669-675, doi: 10.1111/j.1365-2354.2009.01102.x, indexed in Pubmed: 20030691.

9. Nakasono M, Hirokawa M, Muguruma N, et al. Duodenal lymphangitis carcinomatosa: endoscopic characteristics and clinical significance. J Gastroenterol Hepatol. 2006; 21(1 Pt 1): 79-83, doi: 10.1111/j.1440-1746.2005.04203.x, indexed in Pubmed: 16706816.
10. Raja A. Ayloor Seshadri R, Sundersingh S. Lymphangitis Carcinomatosa: Report of a Case and Review of Literature. Indian J Surg Oncol. 2010 Sep. ; 1(3): 274-6.

11. Funakoshi T, Yasui $\mathrm{H}$, Boku N, et al. Clinicopathological features and outcomes of gastric cancer patients with pulmonary lymphangitis carcinomatosa. Jpn J Clin Oncol. 2014; 44(9): 792-798, doi: 10.1093/jjco/hyu091, indexed in Pubmed: 25057093. 